\title{
Second-harmonic generation from realistic film-substrate interfaces: The effects of strain
}

\author{
I. L. Lyubchanskii, ${ }^{\text {a) }}$ N. N. Dadoenkova, and M. I. Lyubchanskii \\ Donetsk Physical-Technical Institute of the National Academy of Sciences of Ukraine, 72, \\ R. Luxemburg str., 340114, Donetsk, Ukraine \\ Th. Rasing \\ Research Institute for Materials, University of Nijmegen, 6525 ED Nijmegen, The Netherlands
}

Jae-Woo Jeong and Sung-Chul Shin ${ }^{\text {b) }}$

Physics Department and CNSM, Korea Advanced Institute of Science and Technology, 373-1 Kusong-dong, Yusung-gu Taejon, 305-701, Korea

(Received 24 June 1999; accepted for publication 5 February 2000)

\begin{abstract}
The optical second-harmonic generation from a thin crystalline film on a substrate is theoretically investigated for both $s$ and $p$ polarized incident light. The contributions of lattice misfit strain as well as of misfit dislocation strain to the second-order nonlinear optical susceptibility are described using a nonlinear photoelastic tensor and can be separated by a polarization analysis of the scattered light at the second harmonic frequency. For the $s(\omega) \rightarrow s(2 \omega)$ and $p(\omega) \rightarrow s(2 \omega)$ scattering geometries, the nonlinear optical signal will be determined by dislocation strain only, whereas for the $s(\omega) \rightarrow p(2 \omega)$ and $p(\omega) \rightarrow p(2 \omega)$ geometries both lattice misfit strain and misfit dislocation strain will contribute. (C) 2000 American Institute of Physics. [S0003-6951(00)01814-3]
\end{abstract}

Nonlinear optical methods, such as optical secondharmonic generation ( $\mathrm{SHG}$ ), are very sensitive to the characteristics of surface and interface structures and have therefore found wide application for the investigation of thin solid films on substrates as well as of multilayerd structures and superlattices. ${ }^{1,2}$

In epitaxially grown thin film structures elastic strain appears, localized near the interface and induced by a misfit of the lattice constants of film and substrate. ${ }^{3}$ The thickness of such a strained layer can vary within a wide range (from a few to a few tens of nanometers $)^{3}$ and is determined by the elastic parameters of both materials and the misfit $f$ :

$$
f=\frac{a_{f}-a_{s}}{a_{s}} .
$$

In Eq. (1) $a_{f}$ and $a_{s}$ are the lattice constants of film and substrate, respectively. Such a strain can lead to an extra contribution to the nonlinear optical polarization and has been observed via SHG. ${ }^{4}$ Moreover, when a film thickness exceeds the critical thickness $h_{c}$, misfit dislocations will appear $^{3}$ that will also contribute to the nonlinear polarization. The influence of such strains and defects on SHG was studied both theoretically and experimentally for semiconductor films and superlattices. ${ }^{4-10}$ The nonzero components of the second-order nonlinear optical susceptibility tensor $\chi^{(2)}$ were calculated in a semimicroscopic approach, based on straininduced changes of the bonds (orbitals) between atoms near the film interfaces. ${ }^{5,6,8}$ Kulyuk et al. ${ }^{7}$ mentioned the additional contribution of strain to the nonlinear polarization, however, without giving any explicit derivations. Alterna-

\footnotetext{
a) Also at: Physics Department and CNSM, Korea Advanced Institute of Science and Technology, 373-1 Kusong-dong, Yusung-gu, Taejon, 305701 Korea.

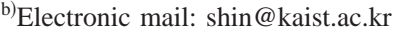

tively, Bottomley ${ }^{9}$ described the influence of dislocation strain on SHG phenomenologically via a periodic modulation of $\chi^{(2)}$ due to displacements of the Bravais lattice sites from their positions in the dislocation-free crystal. ${ }^{9}$ For example, in his description, ${ }^{9}$ contribution along a screw axis dislocation vanishes because the local average symmetry becomes centrosymmetric. This always results in a decrease of the nonlinear optical response, in contrast to experimental observations where strain leads to the appearance of SHG. ${ }^{11}$ In this letter, we propose a more general phenomenological description of SHG from an epitaxially grown film on a substrate, taking into account lattice misfit as well as dislocation strain, by using a nonlinear photoelastic tensor similar to Rayleigh light scattering by dislocation strain. ${ }^{12}$

Consider the interface between a thin crystalline film with cubic symmetry on a thick cubic substrate that is located in the $X Y$ plane with the $Z$ axis perpendicular to this interface (see Fig. 1). A thin film of centrosymmetrical (bulk) material does not contain inversion as an element of the point group symmetry, i.e., the symmetry class of a thin film is determined by its orientation. ${ }^{6,11}$ For example, a thin film of cubic material (point symmetry $O_{h}$ ) with a four-fold axis [001] directed perpendicularly to the film, is characterized by the point symmetry $C_{4 v}$. We investigate a crystal film with thickness $t_{f}$ when $t_{f}>h_{c}$ and misfit dislocations near the interface. The spacing between misfit dislocations $D_{m d}$ is determined by the expression ${ }^{3}$

$$
D_{m d}=\frac{a_{f}}{f} .
$$

The set of equidistant straight edge dislocations oriented along the $Y$ axis and Burgers vectors parallel to the $Y$ axis is 


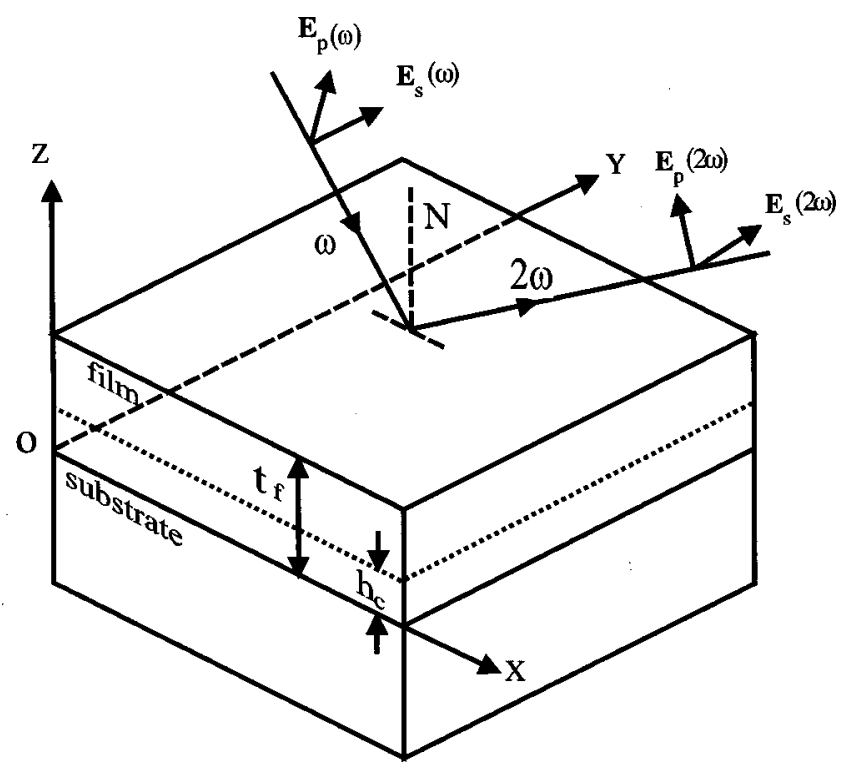

FIG. 1. Schematic image of the second-harmonic generation from a film on a substrate. $N$ is the normal to the film surface.

characterized by the following nonzero components of the stress tensor: ${ }^{13} \sigma_{y y}^{\text {disl }}(\mathbf{r}), \sigma_{z z}^{\text {disl }}(\mathbf{r})$, and $\sigma_{z y}^{\text {disl }}(\mathbf{r})$. The strain tensor in the film can be presented as follows:

$$
u_{l m}(\mathbf{r})=u_{l m}^{\text {misfit }} \theta\left(h_{c}-z\right)+u_{l m}^{\text {disl }}(\mathbf{r}) \theta\left(z-h_{c}\right),
$$

where $\theta(z)$ is the Heaviside step function. The first term in Eq. (3) corresponds to the contribution of the lattice misfit strain and the second one describes the dislocation strain. If the film thickness $t_{f}$ does not exceed the critical thickness $h_{c}$, i.e., $t_{f}<h_{c}$, the film will be free from dislocations and only the first term in Eq. (3) describes the strain in the film. In this case the epilayer will be in a strained state (compressive if $a_{f}>a_{s}$, or tensile if $a_{f}<a_{s}$ ) which is characterized by the biaxial tetragonal strain determined by the expression ${ }^{14}$

$$
u_{l m}^{\text {misfit }}=f \delta_{l m},
$$

where $\delta_{l m}$ is the two-dimensional Kronecker delta symbol (in our case $l, m=x, y$ ). The dislocation strain can be determined using Hooke's law ${ }^{15}$ and the corresponding strain tensor is characterized by the following nonzero components:

$$
\begin{aligned}
& u_{x x}^{\mathrm{disl}}(\mathbf{r})=-\frac{\nu\left[\sigma_{y y}^{\mathrm{disl}}(\mathbf{r})+\sigma_{z z}^{\mathrm{disl}}(\mathbf{r})\right]}{E} \\
& u_{y y}^{\mathrm{disl}}(\mathbf{r})=\frac{\sigma_{y y}^{\mathrm{disl}}(\mathbf{r})-\nu \sigma_{z z}^{\mathrm{disl}}(\mathbf{r})}{E}, \\
& u_{z z}^{\mathrm{disl}}(\mathbf{r})=\frac{\sigma_{z z}^{\mathrm{disl}}(\mathbf{r})-\nu \sigma_{y y}^{\mathrm{disl}}(\mathbf{r})}{E}, \quad u_{z y}^{\mathrm{disl}}(\mathbf{r})=\frac{1+\nu}{E} \sigma_{z y}^{\mathrm{disl}}(\mathbf{r}),
\end{aligned}
$$

where $\nu$ is Poisson's coefficient and $E$ is Young's modulus. ${ }^{15}$

The electric field at the second harmonic frequency of the incident radiation is determined as a solution of the wave equation with the nonlinear polarization $\mathbf{P}^{\mathrm{NL}}(2 \omega)$ as a source term. In the dipole approximation the latter can be written in the well known form ${ }^{1}$

$$
P_{i}^{\mathrm{NL}}(2 \omega)=\chi_{i j k}^{(2)}(-2 \omega: \omega, \omega) E_{j}(\omega) E_{k}(\omega),
$$

where $\mathbf{E}(\omega)$ is the electric field of the incident light at frequency $\omega$ and $\chi^{(2)}$ is the second-order nonlinear optical susDownloaded 13 Jun 2008 to 131.174.20.161. Redistribution subject ceptibility tensor. Like in linear optics, ${ }^{16}$ in the presence of elastic strains this $\chi^{(2)}$ tensor can be presented in the following form:

$$
\chi_{i j k}^{(2)}(\mathbf{r})=\chi_{i j k}^{(2,0)}+p_{i j k l m} u_{l m}(\mathbf{r}) .
$$

Here, $p_{i j k l m}$ and $u_{l m}$ are the nonlinear photoelastic and strain tensors, respectively. Within the slowly varying amplitude approximation the wave equation for the second harmonic electric field can be written as ${ }^{1}$

$2 i k_{2 \omega, l} \nabla_{l} E_{l}(2 \omega, \mathbf{q})=-\frac{\omega^{2}}{c^{2}} \chi_{i j k}^{(2)}(\mathbf{r}) E_{j}(\omega) E_{k}(\omega) \exp (i \mathbf{q} \cdot \mathbf{r})$,

where $\mathbf{q}=2 \mathbf{k}_{\omega}-\mathbf{k}_{2 \omega}$ is the phase (wave vector) mismatch and $\mathbf{k}_{\omega}$ and $\mathbf{k}_{2 \omega}$ are the wave vectors of the fundamental and second harmonic light, respectively. Using the infinite plane wave approximation, ${ }^{1}$ we obtain from Eq. (8)

$$
\begin{aligned}
& E_{i}(2 \omega, \mathbf{q})=\frac{A}{V} \int_{V} \chi_{i j k}^{(2)}(\mathbf{r}) E_{j}(\omega) E_{k}(\omega) \exp (i \mathbf{q r}) d \mathbf{r}, \\
& A=-\frac{i \omega}{c n_{2 \omega}} .
\end{aligned}
$$

The integral in Eq. (9) is taken over the interaction volume $V$ and $n_{2 \omega}$ is the refractive index of the film at the second harmonic frequency.

Let us investigate the polarization of light generated at the double frequency for the wave vectors of the incident and reflected light in the $X Z$ plane (see Fig. 1). For the symmetry class $C_{4 v}$, the second-order optical susceptibility $\chi_{i j k}^{(2,0)}$ (polar third rank) and nonlinear photoelastic $p_{i j k l m}$ (polar fifth rank) tensors are characterized by 4 and 31 independent components, respectively. ${ }^{15}$ Using the nonzero components of the $u_{l m}, \chi_{i j k}^{(2,0)}$, and $p_{i j k l m}$ tensors we can now find the contributions of the misfit strain and dislocation strain to the SHG for $s$ and $p$ polarizations.

(1) $S$-polarized incident light, i.e., $\mathbf{E}(\omega)=\left[0, E_{y}(\omega), 0\right]$ :

$$
\begin{aligned}
E_{x}(2 \omega, \mathbf{q})= & 0, \\
E_{y}(2 \omega, \mathbf{q})= & A p_{y y y z y} u_{z y}(\mathbf{q}) E_{y}^{2}(\omega) \\
E_{z}(2 \omega, \mathbf{q})= & A\left[\chi_{z y y}^{(2,0)} f(\mathbf{q})+p_{z y y x x} u_{x x}(\mathbf{q})+p_{z y y y y} u_{y y}(\mathbf{q})\right. \\
& \left.+p_{z y y z z} u_{z z}(\mathbf{q})\right] E_{y}^{2}(\omega)
\end{aligned}
$$

where

$$
\begin{aligned}
& f(\mathbf{q})=\frac{1}{V} \int_{V} \exp (i \mathbf{q r}) d \mathbf{r}, \\
& u_{l m}(\mathbf{q})=\frac{1}{V} \int_{V} u_{l m}(\mathbf{r}) \exp (i \mathbf{q r}) d \mathbf{r} .
\end{aligned}
$$

Equations $(10 \mathrm{a}-\mathrm{c})$ show that the $s$-polarized electric field Eq. (10b) at the second harmonic frequency is purely determined by the contribution of misfit dislocations. In contrast, the $p$-polarized SHG is determined both by the misfit strain and dislocation strain terms as well as a strain-independent part (10c).

(2) $P$-polarized incident light, i.e., $\mathbf{E}(\omega)$ $=\left[E_{x}(\omega), 0, E_{z}(\omega)\right]:$

\section{西}




$$
\begin{aligned}
E_{x}(2 \omega, \mathbf{q})= & A\left[\left(\chi_{x x z}^{(2,0)}+\chi_{x z x}^{(2,0)}\right) f(\mathbf{q})+\left(p_{x x z x x}+p_{x z x x x}\right)\right. \\
& \times u_{x x}(\mathbf{q})+\left(p_{x x z y y}+p_{x z x y y}\right) u_{y y}(\mathbf{q}) \\
& \left.+\left(p_{x x z z z}+p_{x z x z z}\right) u_{z z}(\mathbf{q})\right] E_{x}(\omega) E_{z}(\omega), \\
E_{y}(2 \omega, \mathbf{q})= & A\left[p_{y x x z y} E_{x}^{2}(\omega)+p_{y z z z y} E_{z}^{2}(\omega)\right] u_{z y}(\mathbf{q}), \\
E_{z}(2 \omega, \mathbf{q})= & A\left\{\left[\chi_{z y y}^{(2,0)} f(\mathbf{q})+p_{z x x x x} u_{x x}(\mathbf{q})+p_{z x x y y} u_{y y}(\mathbf{q})\right.\right. \\
& \left.+p_{z x x z z} u_{z z}(\mathbf{q})\right] E_{x}^{2}(\omega)+\left[\chi_{z z z}^{(2,0)} f(\mathbf{q})\right. \\
& \left.\left.+p_{z z z x x}\left(u_{x x}(\mathbf{q})+u_{y y}(\mathbf{q})\right)\right] E_{z}^{2}(\omega)\right\} .
\end{aligned}
$$

In Eq. (12c) we have taken into account that $p_{z z z x x}$ $=p_{z z z y y} .{ }^{15}$ Equations $(12 \mathrm{a}-\mathrm{c})$ show that for $p$-polarized input, the $s$-polarized second harmonic radiation is determined by dislocation strain only, whereas $p$-polarized output at the second-harmonic frequency is determined by strainindependent as well as strain-dependent terms. It should be noted that both sources of strain (lattice misfit and dislocation) contribute to the $p$-polarized SHG for the $p(\omega)$ $\rightarrow p(2 \omega)$ geometry of scattering. As seen from Eqs. (3)-(5), lattice misfit strain contributes to the SHG via terms which are proportional to $u_{x x}(\mathbf{q})$ and $u_{y y}(\mathbf{q})$, whereas misfit dislocations contribute to the SHG via terms proportional to $u_{x x}(\mathbf{q}), u_{y y}(\mathbf{q}), u_{z y}(\mathbf{q})$, and $u_{z z}(\mathbf{q})$.

As mentioned above, for very thin films with $t_{f}<h_{c}$ only lattice misfit will determine the strain in an epilayer. Then, for both $s$ - and $p$-polarized input light the second-harmonic radiation will be $p$-polarized. This also means that the observation of $s$-polarized SHG will be a clear indication for the appearance of misfit dislocations. It should be noted that for equidistant misfit dislocations the strain-dependent part of $\chi^{(2)}$ can be presented as a periodic function with the period $D_{m d}$, similar to the approach developed in Ref. 9. If this period is comparable with the wavelength of the second harmonic radiation one should expect the appearance of nonlinear diffraction from such a regular dislocation array, similar to the nonlinear diffraction from periodic structures of ferroelectric ${ }^{17}$ or magnetic ${ }^{18}$ domains.

In conclusion, we have presented a phenomenological description of the nonlinear light scattering from a realistic film-substrate interface using nonlinear photoelastic and deformation tensors. This approach allows us to distinguish the contributions of lattice misfit strain and misfit dislocations to the SHG via a polarization analysis of the scattered light.

From experiments ${ }^{19}$ using GaAs in the infrared region with the wavelengths for fundamental and second-harmonic radiation $\lambda_{\omega}=10.6 \mu \mathrm{m}, \lambda_{2 \omega}=5.3 \mu \mathrm{m}$, it was ascertained that the values of nonzero components of the nonlinear photoelastic and second-order nonlinear optical susceptibility tensors were comparable: $\left|p_{x x x y z}\right| \cong 1.2 \times 10^{-10} \mathrm{~m} / \mathrm{V}, \quad \chi_{x y z}^{(2,0)}$ $=1.3 \times 10^{-10} \mathrm{~m} / \mathrm{V}$. Taking into account the values of misfit and dislocation strain, the strain-induced contribution to SHG is estimated about $10 \%$, compared to the strainindependent contribution to SHG.

Results of a more complete theoretical investigation of the angular distribution of second-harmonic radiation scattered by strain in thin film-substrate structures as well as the nonlinear optical diffraction from periodically distributed misfit dislocations will be published elsewhere. A similar analysis of SHG can be applied to magnetic thin films on nonmagnetic substrates. These epitaxially grown multilayer structures will contain a lot of interface strain, as one even manages to grow nonequilibrium structures like facecentered-cubic Fe or body-centered cubic Co (see, for example, Ref. 20). As has been shown in numerous recent publications, SHG is a very sensitive method for the investigation of these magnetic films and interfaces (see, for example, the review article in Ref. 21). In particular the linear magnetization dependence of the SHG signal from magnetic garnet films recently reported by Pavlov et al., ${ }^{22}$ was explained as a result of an interference of crystalline and magnetization-induced terms of the nonlinear optical polarization in these thin films. Such a magnetic-nonmagnetic structure of yttrium-iron garnet grown on a gadoliniumgallium garnet substrate is characterized by a small value of the lattice misfit parameter and a large critical thickness. This leads to a deformed layer near the interface, which also contributes to the nonmagnetic part of the $\chi^{(2)}$ tensor, as was observed in Ref. 22.

This work was supported in part by INTAS, Grant No. 97-0705. One of the authors (I.L.L.) acknowledges KISTEP for financial support for his visit to Korea and is also very grateful to the CNSM and the Physics Department of KAIST for the hospitality extended during his stay in Taejon.

${ }^{1}$ Y. R. Shen, The Principles of Nonlinear Optics (Wiley, New York, 1984).

${ }^{2}$ Proceedings of the Topical Conference: Nonlinear Optics at Interfaces, Berlin, Germany, 1998 [Appl. Phys. B: Lasers Opt. 68, (1999)].

${ }^{3} \mathrm{~J}$. M. Howe, Interfaces in Materials (Wiley-Interscience, New York, 1997).

${ }^{4}$ D. J. Bottomley, A. Mito, P. J. Fons, S. Niki, and A. Yamada, IEEE J. Quantum Electron. 33, 1294 (1997).

${ }^{5}$ V. I. Emelyanov, S. V. Govorkov, N. I. Koroteev, G. I. Petrov, I. L. Schumay, and V. V. Yakovlev, J. Opt. Soc. Am. B 6, 1117 (1989).

${ }^{6}$ S. V. Govorkov, N. I. Koroteev, G. I. Petrov, I. L. Schumay, and V. V. Yakovlev, Appl. Phys. A: Solids Surf. 50, 4439 (1990).

${ }^{7}$ L. L. Kulyuk, D. A. Shutov, E. E. Strubman, and O. A. Aktsipetrov, J. Opt. Soc. Am. B 8, 1766 (1991).

${ }^{8}$ J. V. Huang, Jpn. J. Appl. Phys., Part 1 33A, 3878 (1994).

${ }^{9}$ D. J. Bottomley, Appl. Phys. Lett. 67, 3682 (1995).

${ }^{10}$ C. Zhang, X. Xiao, N. Wang, K. K. Fung, and M. M. T. Loy, Appl. Phys. Lett. 72, 2072 (1998).

${ }^{11}$ R. V. Pisarev, B. B. Krichevtsov, V. N. Gridnev, V. P. Klin, D. Frölich, and Ch. Pahlke-Learch, J. Phys.: Condens. Matter 5, 8621 (1993).

${ }^{12}$ D. Sahoo, A. K. Arora, and R. Kesavamoorthy, J. Phys. C 16, 1687 (1983).

${ }^{13}$ J. P. Hirth and J. Lothe, Theory of Dislocations (McGraw-Hill, New York, 1968).

${ }^{14}$ M. Born and E. Wolf, Principles of Optics (Pergamon, Oxford, 1970).

${ }^{15}$ S. V. Popov, Yu. P. Svirko, and N.I. Zheludev, Ssceptibility Tensors for NonLinear Optics (IOP, Bristol, Philadelphia, 1995).

${ }^{16}$ L. D. Landau and E. M. Lifshitz, Theory of Elasticity (Pergamon, Oxford, 1970).

${ }^{17}$ I. Freund, Phys. Rev. Lett. 21, 1404 (1968).

${ }^{18}$ N. N. Dadoenkova, I. L. Lyubchanskii, M. I. Lyubchanskii, and Th. Rasing, Appl. Phys. Lett. 74, 1880 (1999).

${ }^{19}$ G. D. Boyd, F. R. Nash, and D. F. Nelson, Phys. Rev. Lett. 24, 1298 (1970).

${ }^{20}$ U. Gradmann, in Handbook of Magnetic Materials, edited by K.H.J. Buschow (Elsevier, New York, 1993), Vol. 7.

${ }^{21}$ Th. Rasing, Appl. Phys. B: Lasers Opt. 68, 477 (1999).

${ }^{22}$ V. V. Pavlov, R. V. Pisarev, A. Kirilyuk, and Th. Rasing, Phys. Rev. Lett. 78, 2004 (1997) 